\title{
Spectral Modeling of Switched-Mode Power Converters
}

\author{
C. C. Chan, Fellow, IEEE, and Kwok-Tong Chau, Member, IEEE
}

\begin{abstract}
A new modeling approach for the spectral analysis of pulsewidth modulated (PWM) converters with independent inputs is developed. The key of this approach is to extend the Volterra functional series to nonlinear systems with multiple independent inputs. After formulating the state-space equations describing the dynamical behavior of PWM converters, the Volterra transfer function characterizing the output frequency response can be obtained, which is then symmetrised to form the spectral model. Since the model is developed in a closed form, it is suitable for computer analysis. The modeling approach has been applied to various PWM converters, and the results are verified. The spectral models of different power converters can readily be obtained by using this general approach.
\end{abstract}

\section{INTRODUCTION}

A $S$ the switching operation of power converters turns linear time-invariant systems into nonlinear time-varying systems, the modeling of power converters is one of the major research areas in power electronics. In general, it can be classified as the frequency-domain modeling [1] and the time-domain modeling [2]. The most systematic frequencydomain modeling approach has been that of the state-space averaging [3], which was successfully applied to all pulsewidth modulated (PWM) converters. The use of this technique has been made in deriving an approximated small-signal model of power converters, which provides a tool to access the local stability and is of capital importance in the design of feedback control loop. However, the small-signal modeling can neither handle the large-signal perturbations nor assess the spectral purity of waveforms in power converters. Although the spectral analysis is a well-established tool in signal processing, that finds wide applications in many branches of science and engineering, its application to power electronics is surprisingly little.

In [4], a nonlinear modeling approach was proposed to predict the higher harmonic spectral components of the converter output. The approach simply adopted the Taylor series expansion to model the extracted PWM switch. However, the extraction of the nonlinear switching element from the linear part of the overall system is a rough approximation, and it is also ill-suited to predict the intermodulation spectral components. Due to the presence of output low-pass filters,

Manuscript received April 11, 1992; revised February 26, 1993 and January 14,1994 . This work was supported in part by the Hong Kong Polytechnic under Research Grant 0340.744.A3.410.

C. C. Chan is with the Department of Electrical and Electronic Engineering, University of Hong Kong, Pokfulam Road, Hong Kong.

K. T. Chau is with the Department of Electrical Engineering, Hong Kong Polytechnic, Hung Hom, Kowloon, Hong Kong.

IEEE Log Number 9403300. the low-frequency intermodulation spectral components have more significant effects than the higher harmonic spectral components on the purity of output waveforms.

The Volterra functional series has been used extensively in the spectral analysis of nonlinear circuits and systems. The general theory was mainly developed for single-input nonlinear systems with multiple tones [5]-[7], which were loosely named in [5] as systems with multiple inputs for single-input multitone communication receivers. Hence, the Volterra functional series has recently been applied to the spectral analysis of power converters [8]. However, this spectral analysis has been confined to the output voltage spectrum only contributed by the control signal input, and the spectral contamination contributed by the supply line input has been omitted. In fact, due to the inherent constraint in single-input systems, it is not possible to determine the converter output spectrum contributed simultaneously by the control and line inputs, which are independent of one another [9].

It is the purpose of this paper to newly extend the Volterra functional series to nonlinear systems with multiple independent inputs; in the following, for convenience, they are called multiinput systems. Hence, the spectral modeling of power converters, in the presence of independent inputs, is derived. In order to simplify the subsequent derivation, typical PWM converters (including buck, boost, and buck-boost topologies) are exemplified, where the control signal and line voltage are the independent inputs while the load voltage is the output. The spectral models of different power converters can be obtained by using this general approach.

In applying the Volterra functional series to power converters, the converter is firstly represented by a nonlinear largesignal continuous-time model using the state-space averaging technique. From this model, the output frequency response can be characterized by the Volterra transfer function. The converter spectral model can then be expressed in terms of the symmetrised Volterra transfer function. Moreover, various types of spectral contamination, such as the higher harmonic and intermodulation components, can be individually identified and determined. It should be noted that rather than using the general term of intermodulation as in single-input systems [5]-[8], it is divided into the terms of self-intermodulation and cross-intermodulation for multiinput systems. The former one is due to the intermodulation between tones of each input while another one is due to tones of different inputs.

Since the theory and properties of Volterra functional series for single-input systems have been described in [5]-[8], only a brief overview is given in Section II. Then the Volterra 
functional series is extended to two-input systems, and finally generalized to $m$-input systems. The output frequency response of multiinput multitone nonlinear systems is discussed in Section III. The spectral model of PWM converters is derived in Section IV. Finally, the proposed approach is exemplified and verified in Section V.

\section{Volterra Functional SeRIES}

Volterra first studied the functional series named after him in 1880's as a generalization of the Taylor series expansion of a function [10]. Volterra's book was a summary on its application to the study of certain integral and integro-differential equations [11]. The first application of the Volterra theory to the analysis of a nonlinear device was by Wiener in 1942 [12]. However, the first systematic study of the application of the Volterra functional series to nonlinear systems was by Barrett in 1957 [13]. The development of the Volterra theory of nonlinear systems has led to an extensive study of its application to practical problems in many fields of science and engineering such as system identification [14], circuits [15], antennas [16], communications [17], machines [18], fluid mechanics [19], biophysics [20], and physiology [21]. Nevertheless, its application to problems in electrical engineering has been concentrated on single-input multitone nonlinear systems. In this section, the application of the Volterra functional series is extended to multiinput multitone nonlinear systems.

\section{A. Single-Input Systems}

For single-input analytic systems, the output $y(t)$ can be expressed as a Volterra functional series of the input $a(t)$. Thus

$$
\begin{aligned}
& y(t)=\sum_{n=0}^{\infty} y_{n}(t) \\
& y_{n}(t)= \\
& \qquad \begin{cases}h_{0} & n=0 \\
\int_{-\infty}^{\infty} \cdots \int_{-\infty}^{\infty} h_{n}\left(\tau_{1}, \tau_{2}, \cdots, \tau_{n}\right) \prod_{i=1}^{n} a\left(t-\tau_{i}\right) d \tau_{i} & n>0\end{cases}
\end{aligned}
$$

where $y_{n}(t)$ is called the $n$-th order output of the system, and $h_{n}\left(\tau_{1}, \tau_{2}, \cdots, \tau_{n}\right)$ is called the $n$-th order Volterra kernel of the system. Its multiple Laplace transform

$$
\begin{aligned}
& H_{n}\left(s_{1}, s_{2}, \cdots, s_{n}\right) \\
& = \begin{cases}H_{0}=h_{0} & n=0 \\
\int_{-\infty}^{\infty} \cdots \int_{-\infty}^{\infty} h_{n}\left(\tau_{1}, \tau_{2}, \cdots, \tau_{n}\right) e^{-s_{1} \tau_{1}-s_{2} \tau_{2} \cdots s_{n} \tau_{n}} & \\
\cdot d \tau_{1} d \tau_{2} \cdots d \tau_{n} & n>0\end{cases}
\end{aligned}
$$

is called the $n$-th order Volterra transfer function of the system. Hence, the Volterra model of single-input nonlinear systems is shown in Fig. 1.

Notice that the $n$th order kernel, hence also its transform, is not unique in the sense that several distinct $n$th order kernels may give the same $n$th order output for the same

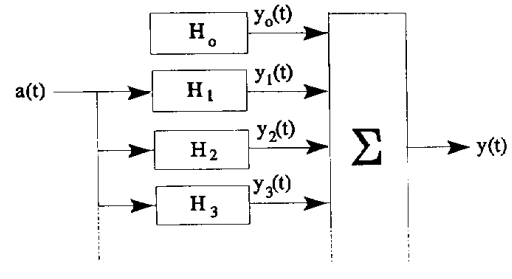

Fig. 1. Volterra model of single-input nonlinear systems.

input. However, the symmetrised kernel and its symmetrised transform defined by

$$
\begin{aligned}
\bar{h}_{n}\left(\tau_{1}, \tau_{2}, \cdots, \tau_{n}\right) & \triangleq \frac{1}{n !} \sum_{\rho\left(\tau_{1}, \tau_{2}, \cdots, \tau_{n}\right)} h_{n}\left(\tau_{1}, \tau_{2}, \cdots, \tau_{n}\right) \\
\bar{H}_{n}\left(s_{1}, s_{2}, \cdots, s_{n}\right) & \triangleq \frac{1}{n !} \sum_{\rho\left(s_{1}, s_{2}, \cdots, s_{n}\right)} H_{n}\left(s_{1}, s_{2}, \cdots, s_{n}\right)
\end{aligned}
$$

are unique, where $\rho(\cdot)$ denotes all permutations of the arguments. Hence, one can freely manipulate them without questioning the validity of such mathematical operations as addition, multiplication, and differentiation, as well as other more complex system operations such as cascading one system into another. Since all kernels can readily be symmetrised, in the following, they will be assumed to be symmetric unless otherwise noted and the overbar will be omitted.

\section{B. Multiinput Systems}

The aforementioned approach is herewith extended to systems with more than one input. As it will be realized, such an extension is very desirable in the case of power electronics systems. Since the first few terms of Volterra functional series are usually sufficient to represent the output as long as the nonlinearity of the system is not too violent, such as the system discussed in this paper, only the first three orders are derived. The higher order outputs can similarly be derived with ever increasing tedium by using the same procedure.

Firstly, a system with two inputs $a(t)$ and $b(t)$ and one output $y(t)$ is considered. The inputs are independent multitone signals. Then proceeding similar to the single-input case, the first few terms of (1) are given by

$$
y_{0}(t)=h_{0}
$$

$$
\begin{aligned}
y_{1}(t) & =y_{1}^{a}(t)+y_{1}^{b}(t) \\
& =\int_{-\infty}^{\infty} h_{1}^{a}(\tau) a(t-\tau) d \tau+\int_{-\infty}^{\infty} h_{1}^{b}(\tau) b(t-\tau) d \tau
\end{aligned}
$$

$$
\begin{aligned}
y_{2}(t)= & y_{2}^{a a}(t)+y_{2}^{a b}(t)+y_{2}^{b b}(t) \\
= & \int_{-\infty}^{\infty} \int_{-\infty}^{\infty} h_{2}^{a a}\left(\tau_{1}, \tau_{2}\right) a\left(t-\tau_{1}\right) a\left(t-\tau_{2}\right) d \tau_{1} d \tau_{2} \\
& +\int_{-\infty}^{\infty} \int_{-\infty}^{\infty} h_{2}^{a b}\left(\tau_{1}, \tau_{2}\right) a\left(t-\tau_{1}\right) b\left(t-\tau_{2}\right) d \tau_{1} d \tau_{2} \\
& +\int_{-\infty}^{\infty} \int_{-\infty}^{\infty} h_{2}^{b b}\left(\tau_{1}, \tau_{2}\right) b\left(t-\tau_{1}\right) b\left(t-\tau_{2}\right) d \tau_{1} d \tau_{2}
\end{aligned}
$$




$$
\begin{aligned}
y_{3}(t)= & y_{3}^{a a a}(t)+y_{3}^{a a b}(t)+y_{3}^{a b b}(t)+y_{3}^{b b b}(t) \\
= & \int_{-\infty}^{\infty} \int_{-\infty}^{\infty} \int_{-\infty}^{\infty} h_{3}^{a a a}\left(\tau_{1}, \tau_{2}, \tau_{3}\right) a\left(t-\tau_{1}\right) a\left(t-\tau_{2}\right) \\
& \times a\left(t-\tau_{3}\right) d \tau_{1} d \tau_{2} d \tau_{3} \\
& +\int_{-\infty}^{\infty} \int_{-\infty}^{\infty} \int_{-\infty}^{\infty} h_{3}^{a a b}\left(\tau_{1}, \tau_{2}, \tau_{3}\right) a\left(t-\tau_{1}\right) a\left(t-\tau_{2}\right) \\
& \times b\left(t-\tau_{3}\right) d \tau_{1} d \tau_{2} d \tau_{3} \\
& +\int_{-\infty}^{\infty} \int_{-\infty}^{\infty} \int_{-\infty}^{\infty} h_{3}^{a b b}\left(\tau_{1}, \tau_{2}, \tau_{3}\right) a\left(t-\tau_{1}\right) b\left(t-\tau_{2}\right) \\
& \times b\left(t-\tau_{3}\right) d \tau_{1} d \tau_{2} d \tau_{3} \\
& +\int_{-\infty}^{\infty} \int_{-\infty}^{\infty} \int_{-\infty}^{\infty} h_{3}^{b b b}\left(\tau_{1}, \tau_{2}, \tau_{3}\right) b\left(t-\tau_{1}\right) b\left(t-\tau_{2}\right) \\
& \times b\left(t-\tau_{3}\right) d \tau_{1} d \tau_{2} d \tau_{3}
\end{aligned}
$$

where $h_{1}^{a}, h_{1}^{b}, h_{2}^{a a}, h_{2}^{b b}, h_{3}^{a a a}, h_{3}^{b b b}$ and so on describing the individual contribution of each input to the output response are named as the self-kernel while $h_{2}^{a b}, h_{3}^{a a b}, h_{3}^{a b b}$ and so on describing the nonlinear interaction of the two inputs to affect the output response are named as the cross-kernel. The multiple Laplace transform of these kernels are

$$
\begin{gathered}
H_{0}=h_{0} \\
H_{1}^{a}(s)=\int_{-\infty}^{\infty} h_{1}^{a}(\tau) e^{-s \tau} d \tau \\
H_{1}^{b}(s)=\int_{-\infty}^{\infty} h_{1}^{b}(\tau) e^{-s \tau} d \tau \\
H_{2}^{a a}\left(s_{1}, s_{2}\right)=\int_{-\infty}^{\infty} \int_{-\infty}^{\infty} h_{2}^{a a}\left(\tau_{1}, \tau_{2}\right) e^{-s_{1} \tau_{1}-s_{2} \tau_{2}} d \tau_{1} d \tau_{2} \\
H_{2}^{a b}\left(s_{1}, s_{2}\right)=\int_{-\infty}^{\infty} \int_{-\infty}^{\infty} h_{2}^{a b}\left(\tau_{1}, \tau_{2}\right) e^{-s_{1} \tau_{1}-s_{2} \tau_{2}} d \tau_{1} d \tau_{2} \\
H_{2}^{b b}\left(s_{1}, s_{2}\right)=\int_{-\infty}^{\infty} \int_{-\infty}^{\infty} h_{2}^{b b}\left(\tau_{1}, \tau_{2}\right) e^{-s_{1} \tau_{1}-s_{2} \tau_{2}} d \tau_{1} d \tau_{2} \\
H_{3}^{a a a}\left(s_{1}, s_{2}, s_{3}\right)=\int_{-\infty}^{\infty} \int_{-\infty}^{\infty} \int_{-\infty}^{\infty} h_{3}^{a a a}\left(\tau_{1}, \tau_{2}, \tau_{3}\right) \\
H_{3}^{a a b}\left(s_{1}, s_{2}, s_{3}\right)=\int_{-\infty}^{\infty} \int_{-\infty}^{\infty} \int_{-\infty}^{\infty} h_{3}^{a a b}\left(\tau_{1}, \tau_{2}, \tau_{3}\right) \\
H_{3}^{a b b}\left(s_{1}, s_{2}, s_{3}\right)=\int_{-\infty}^{\infty} \int_{-\infty}^{\infty} \int_{-\infty}^{\infty} h_{3}^{a b b}\left(\tau_{1}, \tau_{2}, \tau_{3}\right) \\
H_{3}^{b b b}\left(s_{1}, s_{2}, s_{3}\right)=\int_{-\infty}^{\infty} \int_{-\infty}^{\infty} \int_{-\infty}^{\infty} h_{3}^{b b b}\left(\tau_{1}, \tau_{2}, \tau_{3}\right) \\
\times e^{-s_{1} \tau_{1}-s_{2} \tau_{2}-s_{3} \tau_{3}} d \tau_{1} d \tau_{2} d \tau_{3}
\end{gathered}
$$

and the corresponding Volterra model is shown in Fig. 2.

Similarly, for a system with three inputs $a(t), b(t)$ and $c(t)$, the first-order term has the kernels $\left\{h_{1}^{a}(\tau), h_{1}^{b}(\tau), h_{1}^{c}(\tau)\right\}$, the second-order term has the kernels $\left\{h_{2}^{a a}\left(\tau_{1}, \tau_{2}\right), h_{2}^{b b}\left(\tau_{1}, \tau_{2}\right)\right.$, $\left.h_{2}^{c c}\left(\tau_{1}, \tau_{2}\right), h_{2}^{a b}\left(\tau_{1}, \tau_{2}\right), h_{2}^{a c}\left(\tau_{1}, \tau_{2}\right), h_{2}^{b c}\left(\tau_{1}, \tau_{2}\right)\right\}$, and the 3-rd order term has the kernels $\left\{h_{3}^{a a a}\left(\tau_{1}, \tau_{2}, \tau_{3}\right), h_{3}^{\text {bbb }}\left(\tau_{1}, \tau_{2}, \tau_{3}\right)\right.$, $h_{3}^{c c c}\left(\tau_{1}, \tau_{2}, \tau_{3}\right), h_{3}^{a a b}\left(\tau_{1}, \tau_{2}, \tau_{3}\right), h_{3}^{a b b}\left(\tau_{1}, \tau_{2}, \tau_{3}\right), h_{3}^{a a c}\left(\tau_{1}, \tau_{2}\right.$,

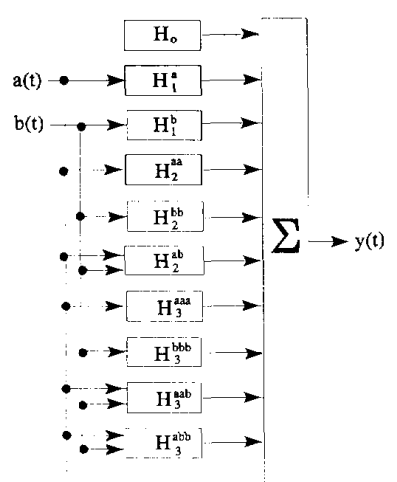

Fig. 2. Volterra model of two-input nonlinear systems.

TABLE I

The First-Order Output Response

\begin{tabular}{llll}
\hline Item & Frequency & Amplitude & Type \\
\hline 1 & $\omega_{21}$ & $\mathrm{~A}_{1} \mathrm{H}_{1}{ }^{2}\left(\mathrm{j} \omega_{\mathrm{al}}\right)$ & Linear \\
2 & $\omega_{22}$ & $\mathrm{~A}_{2} \mathrm{H}_{1}{ }^{\mathrm{a}}\left(\mathrm{j} \omega_{\mathrm{b}}\right)$ & Linear \\
3 & $\omega_{\mathrm{b} 1}$ & $\mathrm{~B}_{1} \mathrm{H}_{1}{ }^{\mathrm{b}}\left(\mathrm{j} \omega_{\mathrm{b}}\right)$ & Linear \\
4 & $\omega_{\mathrm{b2}}$ & $\mathrm{B}_{2} \mathrm{H}_{1}{ }^{\mathrm{b}}\left(\mathrm{j} \omega_{\mathrm{b} 2}\right)$ & Linear \\
\hline
\end{tabular}

$\left.\tau_{3}\right), h_{3}^{a c c}\left(\tau_{1}, \tau_{2}, \tau_{3}\right), h_{3}^{b b c}\left(\tau_{1}, \tau_{2}, \tau_{3}\right), h_{3}^{b c c}\left(\tau_{1}, \tau_{2}, \tau_{3}\right), h_{3}^{a b c}\left(\tau_{1}\right.$, $\left.\left.\tau_{2}, \tau_{3}\right)\right\}$. Hence, for a system with $m$ inputs $\{k i, i=$ $1, \cdots, m\}$, the first-order kernels are $\left\{h_{1}^{k i}, i=1, \cdots, m\right\}$, the second-order kernels are $\left\{h_{2}^{k i k j}, i, j=1, \cdots, m \& i \leq j\right\}$, and the third-order kernels are $\left\{h_{3}^{k i k j k t}, i, j, t=1, \cdots, m \&\right.$ $i \leq j \leq t\}$.

\section{OUTPUT FREQUENCY RESPONSE}

Since the output frequency responses of single-input multitone systems has been described in [5]-[8], in this section, it is interested in discussing the output frequency response of multiinput multitone systems. Firstly, a two-input two-tone nonlinear system is considered. Let

$$
\begin{aligned}
& a(t)=A_{1} \cos \omega_{a 1} t+A_{2} \cos \omega_{a 2} t \\
& b(t)=B_{1} \cos \omega_{b 1} t+B_{2} \cos \omega_{b 2} t
\end{aligned}
$$

be the independent inputs. By substituting (12) into (5) and then using (9), the first-order output response can be determined. Following the same procedure, the second- and the third-order output responses can also be obtained. Due to the nonlinearity of the system, the output response consists of various spectral components: the fundamental, higher harmonic, self-intermodulation and cross-intermodulation components. The first-, the second- and the third-order output responses are derived and tabulated in Tables I, II, and III, respectively. The name of each type of nonlinear responses, as labeled because of its effect in interference studies, is indicated in the last column of the tables.

From Table I, it is obvious that the first-order output response is linearly scaled version of each input because there is no interaction between the tones of the two inputs. 
TABLE II

The Second-Order Output Response

\begin{tabular}{|c|c|c|c|}
\hline Item & Frequency & Amplitude & Type \\
\hline 1 & $2 \omega_{21}$ & $(1 / 2) A_{1}{ }^{2} H_{2}{ }^{2 \mu}\left(j \omega_{n 1}, j \omega_{n 1}\right)$ & Harmonic \\
\hline 2 & $2 \omega_{22}$ & $(1 / 2) A_{2}{ }^{2} H_{2}{ }^{\omega}\left(j \omega_{2}, j \omega_{22}\right)$ & Harmonic \\
\hline 3 & $2 \omega_{b 1}$ & $(1 / 2) \mathrm{B}_{1}{ }^{2} \mathrm{H}_{2}{ }^{\mathrm{bb}}\left(j \omega_{\mathrm{b} 1}, \mathrm{j} \omega_{\mathrm{b} 1}\right)$ & Harmonic \\
\hline 4 & $2 \omega_{\mathrm{b} 2}$ & $(1 / 2) \mathrm{B}_{2}{ }^{2} \mathrm{H}_{2}{ }^{\mathrm{bb}}\left(\mathrm{j} \omega_{\mathrm{b} 2}, \mathrm{j} \omega_{\mathrm{b} 2}\right)$ & Harmonic \\
\hline 5 & $\omega_{n 1}-\omega_{n 1}=0$ & $(1 / 2) \mathrm{A}_{1}^{2} \mathrm{H}_{2}{ }^{\nu}\left(-j \omega_{11}, j \omega_{11}\right)$ & Self-Intermodulation \\
\hline 6 & $\omega_{\mathbf{n} 2}-\omega_{n 2}=0$ & $\left.(1 / 2) A_{2}^{2} H_{2}{ }^{\mu \prime}\left(-j \omega_{2}, j \omega_{22}\right)\right)$ & Self-Intermodulation \\
\hline 7 & $\omega_{n 1}+\omega_{k 2}$ & $A_{1} A_{2} H_{2}{ }^{\omega \alpha}\left(j \omega_{11}, j \omega_{\alpha 2}\right)$ & Self-Intermodulation \\
\hline 8 & $\omega_{11}-\omega_{22}$ & $A_{1} A_{2} H_{2}{ }^{\mu \alpha}\left(j \omega_{s 1},-j \omega_{\alpha 2}\right)$ & Self-Intermodulation \\
\hline 9 & $\omega_{\mathrm{b} 1}-\omega_{\mathrm{b} 1}=0$ & $(1 / 2) B_{1}{ }^{2} H_{2}{ }^{b b}\left(-j \omega_{b 1}, j \omega_{b 1}\right)$ & Self-Intermodulation \\
\hline 10 & $\omega_{\mathrm{b} 2}-\omega_{\mathrm{b} 2}=0$ & $(1 / 2) B_{2}{ }^{2} H_{2}{ }^{b b}\left(-j \omega_{b 2}, j \omega_{b 2}\right)$ & Self-Intermodulation \\
\hline 11 & $\omega_{\mathrm{b} 1}+\omega_{\mathrm{b} 2}$ & $B_{1} B_{2} H_{2}^{B b}\left(j \omega_{b 1}, j \omega_{b 2}\right)$ & Self-Intermodulation \\
\hline 12 & $\omega_{\mathrm{b} 1}-\omega_{\mathrm{b} 2}$ & $\mathrm{~B}_{1} \mathrm{~B}_{2} \mathrm{H}_{2}^{\mathrm{bb}}\left(j \omega_{\mathrm{bl} 1},-j \omega_{\mathrm{b} 2}\right)$ & Self-Intermodulation \\
\hline 13 & $\omega_{\mathrm{a}}+\omega_{\mathrm{b} 1}$ & $(1 / 2) A_{1} B_{1} H_{2}^{a b}\left(j \omega_{a 1}, j \omega_{b 1}\right)$ & Cross-Intermodulation \\
\hline 14 & $\omega_{31}-\omega_{b 1}$ & $(1 / 2) A_{1} B_{1} H_{2}{ }^{a b}\left(j \omega_{a 1},-j \omega_{b 1}\right)$ & Cross-Intermodulation \\
\hline 15 & $\omega_{21}+\omega_{\mathrm{b} 2}$ & $(1 / 2) A_{1} B_{2} H_{2}{ }^{2 b}\left(j \omega_{a 1}, j \omega_{b 2}\right)$ & Cross-Intermodulation \\
\hline 16 & $\omega_{21}-\omega_{b 2}$ & $(1 / 2) A_{1} B_{2} H_{2}^{a b}\left(j \omega_{a 1},-j \omega_{b 2}\right)$ & Cross-Intermodulation \\
\hline 17 & $\omega_{a 2}+\omega_{b 1}$ & $(1 / 2) \mathrm{A}_{2} \mathrm{~B}_{1} \mathrm{H}_{2}{ }^{\star b}\left(\mathrm{j} \omega_{\mathrm{a} 2}, j \omega_{\mathrm{b} 1}\right)$ & Cross-Intermodulation \\
\hline 18 & $\omega_{22}-\omega_{h 1}$ & $(1 / 2) A_{2} B_{1} H_{2}{ }^{a b}\left(j \omega_{a 2},-j \omega_{b 1}\right)$ & Cross-Intermodulation \\
\hline 19 & $\omega_{\mathrm{a} 2}+\omega_{\mathrm{b} 2}$ & $(1 / 2) A_{2} B_{2} H_{2}^{2 b}\left(j \omega_{22}, j \omega_{b 2}\right)$ & Cross-Intermodulation \\
\hline 20 & $\omega_{22}-\omega_{b 2}$ & $(1 / 2) A_{2} B_{2} H_{2}{ }^{a b}\left(j \omega_{22},-j \omega_{b 2}\right)$ & Cross-Intermodulation \\
\hline
\end{tabular}

For the second-order response listed in Table II, items 1-4 are the second harmonic components, items 5-12 are the second-order self-intermodulation components contributed by two tones of each input, and items 13-20 are the secondorder cross-intermodulation components contributed by two tones of different inputs. It can be found that items 5, 6, 9 , and 10 are dc components, which can contribute an additional positive or negative dc offset to the output. Similar to the second-order response, the third-order response consists of the third harmonic, self-intermodulation and cross-intermodulation components. It should be noted that both self- and crossintermodulation may contribute to the fundamental frequency components $\left\{\omega_{a 1}, \omega_{a 2}, \omega_{b 1}, \omega_{b 2}\right\}$; for example, items $9,11,57$, and 58 have contributions at $\omega_{a 1}$. However, these higher order contributions are usually of much smaller amplitude when compared with the lowest order contribution at a particular frequency.

In general, for a system with $m$ inputs $\{k i, i=1, \cdots, m\}$ and the $i$ th input is composed of $Q_{i}$ tones $\left\{\omega_{i x}, x=\right.$ $\left.1, \cdots, Q_{i}\right\}$, the corresponding output frequency response can similarly be determined with ever increasing tedium by using the above procedure. Thus, the first-order output response consists of the first-order transfer functions $\left\{H_{1}^{k i}, i=\right.$ $1, \cdots, m\}$ and the fundamental frequency components $\left\{\omega_{i x}, i=1, \cdots, m \& x=1, \cdots, Q_{i}\right\}$. Similarly, the secondorder output response has the second-order transfer functions $\left\{H_{2}^{k i k j}, i, j=1, \cdots, m \& i \leq j\right\}$ and various frequency components $\left\{\left|\omega_{i x} \pm \omega_{j y}\right|, i, j=1, \cdots, m, x=1, \cdots, Q_{i}\right.$ $\left.\& y=1, \cdots, Q_{j}\right\}$. The 3 -rd order output response has the 3-rd order transfer functions $\left\{H_{3}^{k i k j k t}, i, j, t=1, \cdots, m \&\right.$ $i \leq j \leq t\}$ and various frequency components $\left\{\mid \omega_{i x} \pm\right.$ $\omega_{j y} \pm \omega_{t z} \mid, i, j, t=1, \cdots, m, x=1, \cdots, Q_{i}, y=1, \cdots, Q_{j} \&$ $\left.z=1, \cdots, Q_{t}\right\}$.

\section{SPectral Models of Power CONVERTERS}

Having extended the Volterra functional series to multiinput multitone nonlinear systems and then derived the output frequency response of two-input two-tone systems, the approach is herewith applied to find the spectral model of power converters.

\section{A. Formulation of State-Space Equation}

The first step in deriving the spectral model is to formulate the state-space equation of power converters. There is no doubt that the state-space averaging technique is one of the most systematic methods to obtain a large-signal continuoustime model of the converter. This technique is valid when the natural frequencies of the converter are all well below the switching frequency, which is the case for a practical PWM converter with switching frequencies ranging from tens of kilohertz to hundreds of kilohertz.

The generalized state-space equation for various power converters operating in the continuous conduction mode can be expressed as

$$
\begin{aligned}
\frac{d x}{d t} & =\left[\mu C_{1}+(1-\mu) C_{2}\right] x+\left[\mu D_{1}+(1-\mu) D_{2}\right] u \\
y & =\left[\mu E_{1}+(1-\mu) E_{2}\right] x+\left[\mu F_{1}+(1-\mu) F_{2}\right] u
\end{aligned}
$$

where $\mu$ is the average parameter of the switch, matrices 
TABLE III

The Third-Order Output Response. (a) Harmonic and Self-Intermodulation Components. (b) Cross-Intermodulation Components

\begin{tabular}{|c|c|c|c|}
\hline Item & Frequency & Amplitude & Type \\
\hline 1 & $3 \omega_{11}$ & $(1 / 4) A_{1}{ }^{3} H_{3}{ }^{\cdots}\left(j \omega_{11}, j \omega_{21}, j \omega_{21}\right)$ & Harmonic \\
\hline 2 & $3 \omega_{12}$ & $(1 / 4) A_{2}{ }^{3} H_{3}{ }^{\omega \prime \prime}\left(j \omega_{22}, j \omega_{12}, j \omega_{22}\right)$ & Harmonic \\
\hline 3 & $3 \omega_{\mathrm{bl}}$ & $(1 / 4) B_{1}{ }^{3} H_{3}{ }^{b b b}\left(j \omega_{b 1}, j \omega_{b 1}, j \omega_{b 1}\right)$ & Harmonic \\
\hline 4 & $3 \omega_{\mathrm{b} 2}$ & $(1 / 4) \mathrm{B}_{2}{ }^{3} \mathrm{H}_{3}{ }^{\mathrm{bb}}\left(\mathrm{j} \omega_{\mathrm{b} 2}, \mathrm{j} \omega_{\mathrm{b} 2}, \mathrm{j} \omega_{\mathrm{b} 2}\right)$ & Harmonic \\
\hline 5,6 & $\omega_{21} \pm 2 \omega_{12}$ & $(3 / 4) A_{1} A_{2}^{2} H_{3}{ }^{m-n}\left(j \omega_{11}, \pm j \omega_{22}, \pm j \omega_{a 2}\right)$ & Self-Intermodulation \\
\hline 7,8 & $2 \omega_{a 1} \pm \omega_{12}$ & $\left.(3 / 4) A_{1}^{2} A_{2} H_{3}{ }^{\omega}-j \omega_{a 1}, j \omega_{a 1}, \pm j \omega_{k 2}\right)$ & Self-Intermodulation \\
\hline 9 & $2 \omega_{11}-\omega_{11}=\omega_{11}$ & $\left.(3 / 4) A_{1}{ }^{3} H_{3} \omega_{\left(-j \omega_{11}\right.} j \omega_{11}, j \omega_{11}\right)$ & Self-Intermodulation \\
\hline 10 & $2 \omega_{12}-\omega_{12}=\omega_{12}$ & $(3 / 4) A_{2}{ }^{3} H_{3}{ }^{20}\left(-j \omega_{22}, j \omega_{n 2}, j \omega_{n 2}\right)$ & Self-Intermodulation \\
\hline 11 & $\omega_{\mathbf{1 1}}-\omega_{\mathbf{1 2}}+\omega_{\mathbf{2 2}}=\omega_{\mathbf{1 1}}$ & $(3 / 2) A_{1} A_{2}{ }^{2} H_{3}{ }^{m a}\left(j \omega_{n 1},-j \omega_{n 2}, j \omega_{22}\right)$ & Self-Intermodulation \\
\hline 12 & $-\omega_{\mathrm{n} 1}+\omega_{\mathrm{n} 1}+\omega_{\mathrm{k2}}=\omega_{\mathrm{n} 2}$ & $(3 / 2) A_{1}^{2} A_{2} H_{3}{ }^{\omega}\left(-j \omega_{21}, j \omega_{21}, j \omega_{22}\right)$ & Self-Intermodulation \\
\hline 13,14 & $\omega_{b 1} \pm 2 \omega_{b 2}$ & $(3 / 4) B_{1} B_{2}{ }^{2} H_{3}{ }^{b b b}\left(j \omega_{b 1}, \pm j \omega_{b 2}, \pm j \omega_{b 2}\right)$ & Self-Intermodulation \\
\hline 15,16 & $2 \omega_{b 1} \pm \omega_{b 2}$ & $(3 / 4) B_{1}{ }^{2} B_{2} H_{3}^{b b b}\left(j \omega_{b 1}, j \omega_{b 1}, \pm j \omega_{b 2}\right)$ & Self-Intermodulation \\
\hline 17 & $2 \omega_{b 1}-\omega_{b 1}=\omega_{b 1}$ & $(3 / 4) B_{1}{ }^{3} H_{3}{ }^{b b b}\left(-j \omega_{b 1}, j \omega_{b 1}, j \omega_{b 1}\right)$ & Self-Intermodulation \\
\hline 18 & $2 \omega_{\mathrm{b} 2}-\omega_{\mathrm{b} 2}=\omega_{\mathrm{b} 2}$ & $(3 / 4) B_{2}{ }^{3} H_{3}{ }^{b b b}\left(-j \omega_{b 2}, j \omega_{b 2}, j \omega_{b 2}\right)$ & Self-Intermodulation \\
\hline 19 & $\omega_{b 1}-\omega_{b 2}+\omega_{b 2}=\omega_{b 1}$ & $(3 / 2) B_{1} B_{2}{ }^{2} H_{3}{ }^{b b b}\left(j \omega_{b 1},-j \omega_{b 2}, j \omega_{b 2}\right)$ & Self-Intermodulation \\
\hline 20 & $-w_{b 1}+\omega_{b 1}+w_{b 2}=\omega_{b 2}$ & $(3 / 2) B_{1}^{2} B_{2} H_{3}^{b b b}\left(-j \omega_{b 1}, j \omega_{b 1}, j \omega_{b 2}\right)$ & Self-Intermodulation \\
\hline
\end{tabular}

(a)

\begin{tabular}{|c|c|c|c|}
\hline Item & Frequency & Amplitude & Type \\
\hline 21,22 & $2 \omega_{21} \pm \omega_{b 1}$ & $(1 / 4) A_{1}{ }^{2} B_{1} H_{3}{ }^{\omega b}\left(j \omega_{1}, j \omega_{a l}, \pm j \omega_{b 1}\right)$ & Cross-Intermodulation \\
\hline 23,24 & $2 \omega_{a 1} \pm \omega_{b 2}$ & $(1 / 4) A_{1}{ }^{2} B_{2} H_{3}{ }^{\omega s}\left(j \omega_{1}, j \omega_{a 1}, \pm j \omega_{b 2}\right)$ & Cross-Intermodulation \\
\hline 25,26 & $2 \omega_{a} \pm \omega_{b t}$ & $(1 / 4) A_{2}{ }^{2} B_{1} H_{3}{ }^{\mu b}\left(j \omega_{2,}, j \omega_{22}, \pm j \omega_{b 1}\right)$ & Cross-Intermodulation \\
\hline 27,28 & $2 \omega_{n_{2}} \pm \omega_{b 2}$ & $(1 / 4) A_{2}{ }^{2} B_{2} H_{3}{ }^{a b}\left(j \omega_{12}, j \omega_{a 2}, \pm j \omega_{b 2}\right)$ & Cross-Intermodulation \\
\hline 29,30 & $\omega_{21} \pm \omega_{22}+\omega_{b 1}$ & $(1 / 2) A_{1} A_{2} B_{1} H_{3}{ }^{\omega b}\left(j \omega_{a 1}, \pm j \omega_{2}, j \omega_{b 1}\right)$ & Cross-Intermodulation \\
\hline 31,32 & $\omega_{a 1} \pm \omega_{22}+\omega_{b 2}$ & $(I / 2) A_{1} A_{2} B_{2} H_{3}{ }^{a b}\left(j \omega_{a 1}, \pm j \omega_{a 2}, j \omega_{b 2}\right)$ & Cross-Intermodulation \\
\hline 33,34 & $\omega_{\mathrm{a} 1} \pm \omega_{\mathrm{n} 2}-\omega_{\mathrm{b} 1}$ & $(1 / 2) A_{1} A_{2} B_{1} H_{3}{ }^{a b}\left(j \omega_{a 1}, \pm j \omega_{2},-j \omega_{b 1}\right)$ & Cross-Intermodulation \\
\hline 35,36 & $\omega_{11} \pm \omega_{22}-\omega_{b 2}$ & $(1 / 2) A_{1} A_{2} B_{2} H_{3}^{a a b}\left(j \omega_{11}, \pm j \omega_{a 2},-j \omega_{b 2}\right)$ & Cross-Intermodulation \\
\hline 37 & $\omega_{\mathrm{Al}}-\omega_{\mathrm{11}}+\omega_{\mathrm{bl}}=\omega_{\mathrm{bl}}$ & $(1 / 2) A_{1}^{2} B_{1} H_{3}{ }^{\omega b}\left(-j \omega_{21}, j \omega_{21}, j \omega_{b 1}\right)$ & Cross-Intermodulation \\
\hline 38 & $\omega_{\mathrm{a}_{2}-}-\omega_{\mathrm{a} 2}+\omega_{\mathrm{bl}}=\omega_{\mathrm{bl}}$ & $(1 / 2) A_{2}{ }^{2} B_{1} H_{3}{ }^{\Delta b}\left(-j \omega_{22}, j \omega_{a 2}, j \omega_{b 1}\right)$ & Cross-Intermodulation \\
\hline 39 & $\omega_{\mathrm{a} 1}-\omega_{\mathrm{n} 1}+\omega_{\mathrm{b} 2}=\omega_{\mathrm{b} 2}$ & $(1 / 2) A_{1}^{2} B_{2} H_{3}{ }^{m b}\left(-j \omega_{a 1}, j \omega_{a 1}, j \omega_{b 2}\right)$ & Cross-Intermodulation \\
\hline 40 & $\omega_{\mathrm{n} 2}-\omega_{\mathrm{n} 2}+\omega_{\mathrm{b} 2}=\omega_{\mathrm{b} 2}$ & $(1 / 2) A_{2}^{2} B_{2} H_{3}^{a b}\left(-j \omega_{a 2}, j \omega_{22}, j \omega_{b 2}\right)$ & Cross-Intermodulation \\
\hline 41,42 & $\omega_{a 1} \pm 2 \omega_{b 1}$ & $(1 / 4) A_{1} B_{1}{ }^{2} H_{3}{ }^{a b b}\left(j \omega_{21}, \pm j \omega_{b 1}, \pm j \omega_{b 1}\right)$ & Cross-Intermodulation \\
\hline 43,44 & $\omega_{21} \pm 2 \omega_{b 2}$ & $(1 / 4) A_{1} B_{2}^{2} H_{3}{ }^{a b b}\left(j \omega_{a 1}, \pm j \omega_{b 2}, \pm j \omega_{b 2}\right)$ & Cross-Intermodulation \\
\hline 45,46 & $\omega_{22} \pm 2 \omega_{b 1}$ & $(1 / 4) A_{2} B_{1}{ }^{2} H_{3}{ }^{\Delta b}\left(j \omega_{22}, \pm j \omega_{b 1}, \pm j \omega_{b 1}\right)$ & Cross-Intermodulation \\
\hline 47,48 & $\omega_{a 2} \pm 2 \omega_{b 2}$ & $(1 / 4) A_{2} B_{2}{ }^{2} H_{3}{ }^{a b o}\left(j \omega_{22}, \pm j \omega_{b 2}, \pm j \omega_{b 2}\right)$ & Cross-Intermodulation \\
\hline 49,50 & $\omega_{11}+\omega_{b 1} \pm \omega_{b 2}$ & $(1 / 2) A_{1} B_{1} B_{2} H_{3}{ }^{a b}\left(j \omega_{21}, j \omega_{b 1}, \pm j \omega_{b 2}\right)$ & Cross-Intermodulation \\
\hline 51,52 & $\omega_{\mathrm{a} 1}-\omega_{\mathrm{b} 1} \pm \omega_{\mathrm{b} 2}$ & $(1 / 2) A_{1} B_{1} B_{2} H_{3}{ }^{\circ b b}\left(j \omega_{21},-j \omega_{b 1}, \pm j \omega_{b 2}\right)$ & Cross-Intermodulation \\
\hline 53,54 & $\omega_{\mathrm{a} 2}+\omega_{\mathrm{b} 1} \pm \omega_{\mathrm{b} 2}$ & $(1 / 2) A_{2} B_{1} B_{2} H_{3}^{a b b}\left(j \omega_{22}, j \omega_{b 1}, \pm j \omega_{b 2}\right)$ & Cross-Intermodulation \\
\hline 55,56 & $\omega_{22}-\omega_{h 1} \pm \omega_{b 2}$ & $(1 / 2) A_{2} B_{1} B_{2} H_{3}{ }^{a b b}\left(j \omega_{22},-j \omega_{b 1}, \pm j \omega_{b 2}\right)$ & Cross-Intermodulation \\
\hline 57 & $\omega_{\mathrm{al}}-\omega_{\mathrm{bl}}+\omega_{\mathrm{bl}}=\omega_{\mathrm{al}}$ & $(1 / 2) A_{1} B_{1}^{2} H_{3}{ }^{a b b}\left(j \omega_{a 1},-j \omega_{b 1}, j \omega_{b 1}\right)$ & Cross-Intermodulation \\
\hline 58 & $\omega_{\mathrm{a} 1}-\omega_{\mathrm{b2} 2}+\omega_{\mathrm{b} 2}=\omega_{\mathrm{a} 1}$ & $(1 / 2) A_{1} B_{2}^{2} H_{3}{ }^{a b b}\left(j \omega_{11},-j \omega_{b 2}, j \omega_{b 2}\right)$ & Cross-Intermodulation \\
\hline 59 & $\omega_{\mathrm{a}_{2}-}-\omega_{\mathrm{b} 1}+\omega_{\mathrm{b} 1}=\omega_{22}$ & $(1 / 2) A_{2} B_{1}^{2} H_{3}^{a b b}\left(j \omega_{22},-j \omega_{b 1}, j \omega_{b 1}\right)$ & Cross-Intermodulation \\
\hline 60 & $\omega_{\mathrm{a} 2}-\omega_{\mathrm{b} 2}+\omega_{\mathrm{b} 2}=\omega_{\mathrm{a} 2}$ & $(1 / 2) A_{2} B_{2}{ }^{2} H_{3}{ }^{a b b}\left(j \omega_{n 2},-j \omega_{b 2}, j \omega_{b 2}\right)$ & Cross-Intermodulation \\
\hline
\end{tabular}

(b)

$C_{1}, C_{2}, D_{1}, D_{2}, E_{1}, E_{2}, F_{1}$, and $F_{2}$ are all functions of the converter topology, $x$ is the state vector, $u$ is the excitation, and $y$ is the output [22]. For PWM converters, the average parameter of the switch is the duty cycle $\delta$, the excitation is the supply line voltage $v_{g}$, and the matrices $F_{1}$ and $F_{2}$ become zero. The corresponding state-space equations are rewritten as

$$
\begin{aligned}
\frac{d x}{d t} & =\left[\delta C_{1}+(1-\delta) C_{2}\right] x+\left[\delta D_{1}+(1-\delta) D_{2}\right] v_{g} \\
v_{0} & =\left[\partial E_{1}+(1-\partial) E_{2}\right] x
\end{aligned}
$$

where $v_{0}$ is the output voltage across the resistive load. 
Perturbations in $\delta$ and $v_{g}$ causes perturbations in $x$ and $v_{0}$. Thus

$$
\begin{aligned}
\delta & =\bar{\delta}+\tilde{\delta} \\
v_{g} & =\bar{v}_{g}+\tilde{v}_{g} \\
x & =\bar{x}+\tilde{x} \\
v_{0} & =\bar{v}_{0}+\tilde{v}_{0}
\end{aligned}
$$

where steady-state and perturbed quantities are indicated with a bar and a tilde, respectively. By substituting (15) into (14) and then separating the steady-state and perturbed quantities, the state-space equation describing the steady-state behavior of PWM converters can be expressed as

$$
\begin{aligned}
\frac{d \bar{x}}{d t} & =\left[\bar{\delta} C_{1}+(1-\bar{\delta}) C_{2}\right] \bar{x}+\left[\bar{\delta} D_{1}+(1-\bar{\delta}) D_{2}\right] \bar{v}_{g}=0 \\
\bar{v}_{0} & =\left[\bar{\partial} E_{1}+(1-\bar{\partial}) E_{2}\right] \bar{x}
\end{aligned}
$$

and the following state-space equation describing its dynamical behavior

$$
\begin{aligned}
\frac{d \tilde{x}}{d t} & =K_{1} \tilde{x}+K_{2} \tilde{\delta}+K_{3} \tilde{v}_{g}+K_{4} \tilde{x} \tilde{\delta}+K_{5} \tilde{\delta} \tilde{v}_{g} \\
\tilde{v}_{0} & =K_{6} \tilde{x}
\end{aligned}
$$

where

$$
\begin{aligned}
K_{1} & =\bar{\delta} C_{1}+(1-\bar{\delta}) C_{2} \\
K_{2} & =\left(C_{1}-C_{2}\right) \bar{x}+\left(D_{1}-D_{2}\right) \bar{v}_{g} \\
K_{3} & =\bar{\delta} D_{1}+(1-\bar{\delta}) D_{2} \\
K_{4} & =C_{1}-C_{2} \\
K_{5} & =D_{1}-D_{2} \\
K_{6} & =\bar{\delta} E_{1}+(1-\bar{\delta}) E_{2}
\end{aligned}
$$

can be obtained. It should be noted that the product terms with coefficients $K_{4}$ and $K_{5}$ in (17) represent the nonlinearity of PWM converters.

\section{B. Determination of Transfer Functions}

Having derived the state-space equation describing the dynamical behavior of PWM converters, the first-, the secondand the third-order Volterra transfer functions can be determined. A convenient method of evaluating these transfer functions is the so-called "probing" or "harmonic input" method [5]. The system is first "probed" by two singleexponential inputs

$$
\begin{aligned}
\tilde{\delta}(t) & =a(t)=e^{s_{a} t} \\
\tilde{v}_{g}(t) & =b(t)=e^{s_{b} t}
\end{aligned}
$$

and the state vector is expressed as

$$
\tilde{x}=G_{1}^{a}\left(s_{a}\right) e^{s_{a} t}+G_{1}^{b}\left(s_{b}\right) e^{s_{b} t}+\cdots
$$

where $G_{1}^{a}\left(s_{a}\right)$ and $G_{1}^{b}\left(s_{b}\right)$ are to be determined. The terms hidden in the ellipsis in (20) have no contribution to the terms of interest and are omitted. By substituting (19) into (5) and then using (9), it can be found that the coefficients of $e^{s_{a} t}$ and $e^{s_{b} t}$ are $H_{1}^{a}\left(s_{a}\right)$ and $H_{1}^{b}\left(s_{b}\right)$, respectively. Hence, using (17) and $(20)$, the following relationship

$$
\begin{gathered}
H_{1}^{a}\left(s_{a}\right)=K_{6} G_{1}^{a}\left(s_{a}\right) \\
H_{1}^{b}\left(s_{b}\right)=K_{6} G_{1}^{b}\left(s_{b}\right)
\end{gathered}
$$

can be obtained. By substituting (19) and (20) into (17) and then equating the coefficients of $e^{s_{a} t}$ and $e^{s_{b} t}, G_{1}^{a}\left(s_{a}\right)$ and $G_{1}^{b}\left(s_{b}\right)$ can be determined respectively. Thus (21) can be rewritten as

$$
\begin{aligned}
& H_{1}^{a}(s)=K_{6}\left(s I-K_{1}\right)^{-1} K_{2} \\
& H_{1}^{b}(s)=K_{6}\left(s I-K_{1}\right)^{-1} K_{3}
\end{aligned}
$$

which are the first-order transfer functions.

Proceeding similarly with two two-exponential inputs

$$
\begin{aligned}
\tilde{\delta}(t) & =a(t)=e^{s_{a 1} t}+e^{s_{a 2} t} \\
\tilde{v}_{g}(t) & =b(t)=e^{s_{b 1} t}+e^{s_{b 2} t}
\end{aligned}
$$

and the resulted expressions

$$
\begin{aligned}
H_{2}^{a a}\left(s_{1}, s_{2}\right)= & K_{6}\left[\left(s_{1}+s_{2}\right) I-K_{1}\right]^{-1} K_{4}\left(s_{1} I-K_{1}\right)^{-1} K_{2} \\
H_{2}^{a b}\left(s_{1}, s_{2}\right)= & K_{6}\left[\left(s_{1}+s_{2}\right) I-K_{1}\right]^{-1} \\
& \times\left[K_{4}\left(s_{2} I-K_{1}\right)^{-1} K_{3}+K_{5}\right] \\
H_{2}^{b b}\left(s_{1}, s_{2}\right)= & 0
\end{aligned}
$$

are the second-order transfer functions. Following the same procedure with two three-exponential inputs, the third-order transfer functions

$$
\begin{aligned}
H_{3}^{a a a}\left(s_{1}, s_{2}, s_{3}\right)= & K_{6}\left[\left(s_{1}+s_{2}+s_{3}\right) I-K_{1}\right]^{-1} \\
& \times K_{4}\left[\left(s_{1}+s_{2}\right) I-K_{1}\right]^{-1} \\
& \times K_{4}\left(s_{1} I-K_{1}\right)^{-1} K_{2} \\
H_{3}^{a a b}\left(s_{1}, s_{2}, s_{3}\right)= & K_{6}\left[\left(s_{1}+s_{2}+s_{3}\right) I-K_{1}\right]^{-1} \\
& \times K_{4}\left[\left(s_{1}+s_{3}\right) I-K_{1}\right]^{-1} \\
& \times\left[K_{4}\left(s_{3} I-K_{1}\right)^{-1} K_{3}+K_{5}\right] \\
H_{3}^{a b b}\left(s_{1}, s_{2}, s_{3}\right)= & 0 \\
H_{3}^{b b b}\left(s_{1}, s_{2}, s_{3}\right)= & 0
\end{aligned}
$$

can be deduced. It should be noted that $H_{2}^{b b}\left(s_{1}, s_{2}\right)$, $H_{3}^{a b b}\left(s_{1}, s_{2}, s_{3}\right)$, and $H_{3}^{b b b}\left(s_{1}, s_{2}, s_{3}\right)$ are zero because there is no interaction between the tones of $\tilde{v}_{g}$ as given in (17).

Since the resulted transfer functions may not be symmetric, by using (3), the symmetrised nonzero transfer functions are listed as follows

$$
\begin{aligned}
& \bar{H}_{1}^{a}(s)=H_{1}^{a}(s) \\
& \bar{H}_{1}^{b}(s)=H_{1}^{b}(s) \\
\bar{H}_{2}^{a a}\left(s_{1}, s_{2}\right)= & \frac{1}{2 !}\left[H_{2}^{a a}\left(s_{1}, s_{2}\right)+H_{2}^{a a}\left(s_{2}, s_{1}\right)\right] \\
\bar{H}_{2}^{a b}\left(s_{1}, s_{2}\right)= & \frac{1}{2 !}\left[H_{2}^{a b}\left(s_{1}, s_{2}\right)+H_{2}^{a b}\left(s_{2}, s_{1}\right)\right] \\
\bar{H}_{3}^{a a a}\left(s_{1}, s_{2}, s_{3}\right)= & \frac{1}{3 !}\left[H_{3}^{a a a}\left(s_{1}, s_{2}, s_{3}\right)+H_{3}^{a a a}\left(s_{1}, s_{3}, s_{2}\right)\right. \\
& +H_{3}^{a a a}\left(s_{2}, s_{1}, s_{3}\right)+H_{3}^{a a a}\left(s_{2}, s_{3}, s_{1}\right) \\
& \left.+H_{3}^{a a a}\left(s_{3}, s_{1}, s_{2}\right)+H_{3}^{a a a}\left(s_{3}, s_{2}, s_{1}\right)\right] \\
\bar{H}_{3}^{a a b}\left(s_{1}, s_{2}, s_{3}\right)= & \frac{1}{3 !}\left[H_{3}^{a a b}\left(s_{1}, s_{2}, s_{3}\right)+H_{3}^{a a b}\left(s_{1}, s_{3}, s_{2}\right)\right. \\
& +H_{3}^{a a b}\left(s_{2}, s_{1}, s_{3}\right)+H_{3}^{a a b}\left(s_{2}, s_{3}, s_{1}\right) \\
& \left.+H_{3}^{a a b}\left(s_{3}, s_{1}, s_{2}\right)+H_{3}^{a a b}\left(s_{3}, s_{2}, s_{1}\right)\right]
\end{aligned}
$$

which are used to determine the spectral model of PWM converters. 


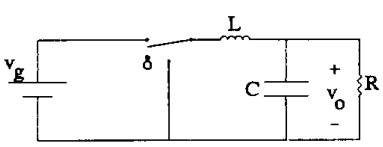

(a)

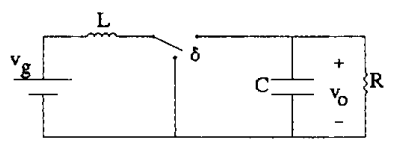

(b)

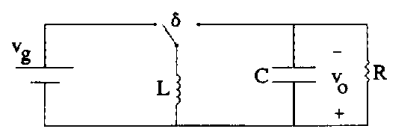

(c)

Fig. 3. 'Typical PWM converters. (a) Buck. (b) Boost. (c) Buck-boost.

TABLE IV

Spectral Components of Buck Converter OutPut Voltage

\begin{tabular}{lll}
\hline Frequency $(\mathrm{H} z)$ & FFT Magnitude $(\mathrm{dB})$ & VFS Magnitude $(\mathrm{dB})$ \\
\hline 0 & 0 & 0 \\
200 & -13.9 & -13.9 \\
300 & -13.9 & -13.9 \\
500 & -27.7 & -27.6 \\
700 & -13.5 & -13.3 \\
800 & -13.3 & -13.2 \\
1000 & -26.8 & -26.7 \\
\hline
\end{tabular}

\section{VERIFICATION}

In order to verify the proposed spectral modeling approach as well as to testify its accuracy, it is compared with the results obtained from PSpice simulation [23]. For a particular case, both of the modeling and simulation results are further compared with the experimental results. Firstly, by using PSpice to perform a tedious startup transient time-domain simulation, the steady-state output waveform can be obtained. Then by applying the Fast Fourier Transform (FFT) to the steady-state waveform, the output spectral components can be determined. Secondly, by using (22) and (24)-(28) as well as Tables I, II, and III, the output spectral components can also be determined. Hence, the FFT results can be numerically compared with the proposed Volterra functional series (VFS) results. It should be noted that the FFT results are obtained with the expense of several hours for the time-domain simulation while the VFS results are obtained within a second. Typical PWM converters are used for exemplification.

\section{A. PWM Buck Converters}

As shown in Fig. 3(a), the PWM buck converter operating at $50 \mathrm{kHz}$ has component values of $L=500 \mu \mathrm{H}, C=10 \mu \mathrm{F}$, and $R=10 \Omega$. The control signal and line voltage

$$
\begin{aligned}
\delta & =0.5+0.1 \cos 2 \pi(700) t+0.1 \cos 2 \pi(800) t \\
v_{g} & =20+4 \cos 2 \pi(200) t+4 \cos 2 \pi(300) t V
\end{aligned}
$$

are the two independent two-tone inputs.

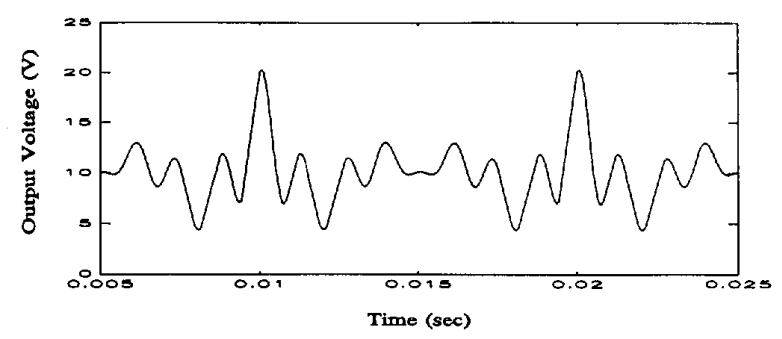

(a)

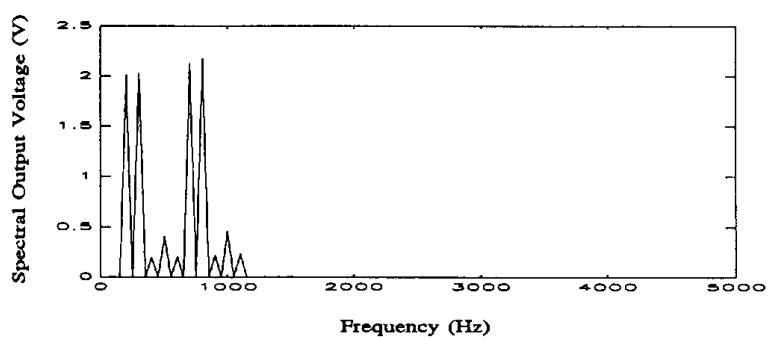

(b)

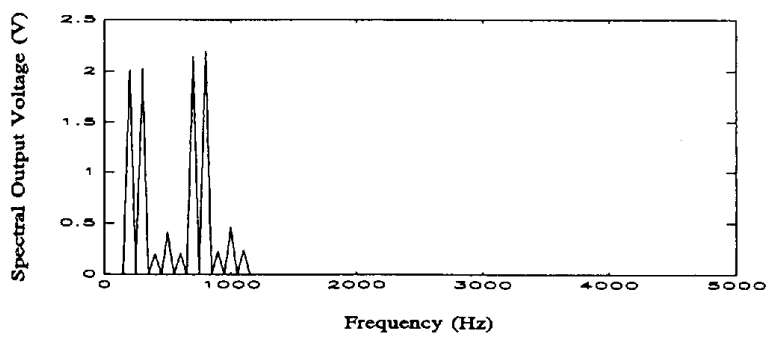

(c)

Fig. 4. Output voltage of buck converter. (a) Waveform using PSpice. (b) Spectrum using FFT. (c) Spectrum using VFS.

The steady-state output voltage waveform resulted from the PSpice simulation is shown in Fig. 4(a). Excluding the dc component, the corresponding FFT spectrum is plotted in Fig. 4(b). By comparing with the spectrum obtained from the proposed approach, namely the VFS spectrum as shown in Fig. 4(c), it can be seen that the VFS spectrum follows the FFT spectrum very closely. Moreover, the relative magnitude of significant spectral components with respect to the dc component is tabulated in Table IV, and the agreement is seen to be very good.

Since $\mathrm{C} 1$ and $\mathrm{C} 2$ in (14) are the same for the PWM buck converter, $K_{4}$ in (17) becomes zero, which implies that there is no interaction between the tones of each input. Apart from the fundamental components, only the second-order interaction between the tones of $\tilde{\delta}$ and $\tilde{g}$ exists. Thus, there is no spectral component at $100 \mathrm{~Hz}$ or above $1100 \mathrm{~Hz}$, which can be observed from Fig. 4(b) or (c).

\section{B. PWM Boost Converters}

The schematic of the PWM boost converter is shown in Fig. 3(b). It is also operated at $50 \mathrm{kHz}$ and has component values of $L=500 \mu \mathrm{H}, C=10 \mu \mathrm{F}$, and $R=10 \Omega$. The 
TABLE V

SPECTRAL Components of Boost CONVERTER OUtPut Voltage

\begin{tabular}{lll}
\hline Frequency $(\mathrm{Hz})$ & FFT Magnitude $(\mathrm{dB})$ & VFS Magnitude $(\mathrm{dB})$ \\
\hline 0 & 0 & 0 \\
200 & -14.1 & -14.1 \\
300 & -14.2 & -14.1 \\
500 & -28.0 & -28.1 \\
700 & -12.5 & -12.6 \\
800 & -12.3 & -12.3 \\
1000 & -25.5 & -25.3 \\
1400 & -26.0 & -25.9 \\
1500 & -21.1 & -20.8 \\
1600 & -26.9 & -26.8 \\
\end{tabular}

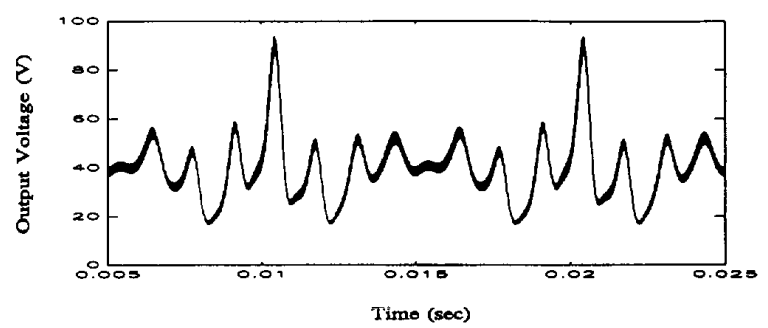

(a)

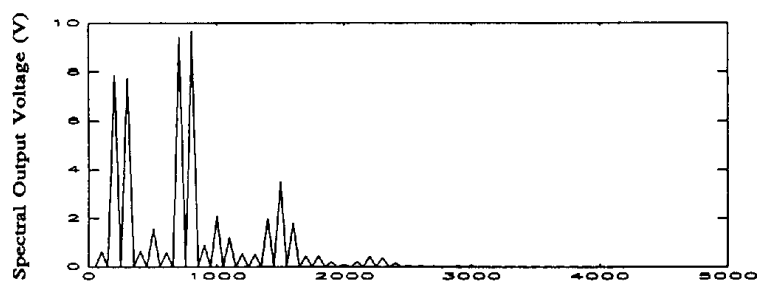

Frequency (Hz)

(b)

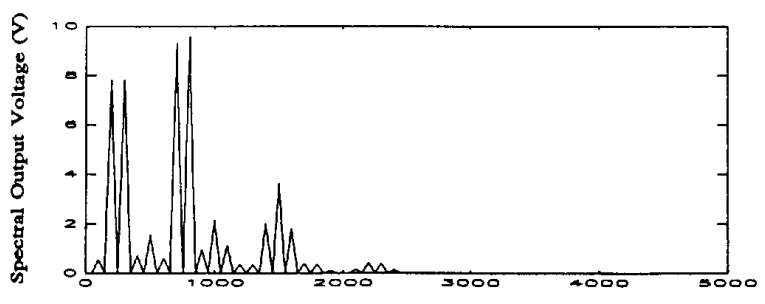

Frequency (Hz)

(c)

Fig. 5. Output voltage of boost converter. (a) Waveform using PSpice. (b) Spectrum using FFT. (c) Spectrum using VFS.

control and line inputs are the same as those of the buck converter given by (29).

Following the same procedure used for the buck converter, the steady-state output voltage waveform is shown in Fig. 5(a), the FFT spectrum is plotted in Fig. 5(b), the VFS spectrum is plotted in Fig. 5(c), and the significant spectral components are tabulated in Table V. As expected, the agreement between the FFT and the VFS results is very good.
TABLE VI

Spectral Components of Boost Amplifier OutPut Load Current

\begin{tabular}{llll}
\hline $\begin{array}{l}\text { Frequency } \\
(\mathrm{Hz})\end{array}$ & $\begin{array}{l}\text { Measurement } \\
(\mathrm{dB})\end{array}$ & $\begin{array}{l}\text { FFT Magnitude } \\
(\mathrm{dB})\end{array}$ & $\begin{array}{l}\text { VFS Magnitude } \\
(\mathrm{dB})\end{array}$ \\
\hline 600 & -27 & -28.0 & -28.2 \\
700 & 0 & -0.4 & -0.4 \\
800 & 0 & 0 & 0 \\
900 & -27 & -27.9 & -28.2 \\
1400 & -17 & -15.9 & -15.9 \\
1500 & -11 & -10.1 & -10.2 \\
1600 & -17 & -16.6 & -16.6 \\
2100 & -39 & -35.5 & -35.5 \\
2200 & -28 & -26.7 & -27.0 \\
2300 & -30 & -28.1 & -28.2 \\
2400 & -41 & -39.0 & -39.1 \\
& & & \\
\hline
\end{tabular}

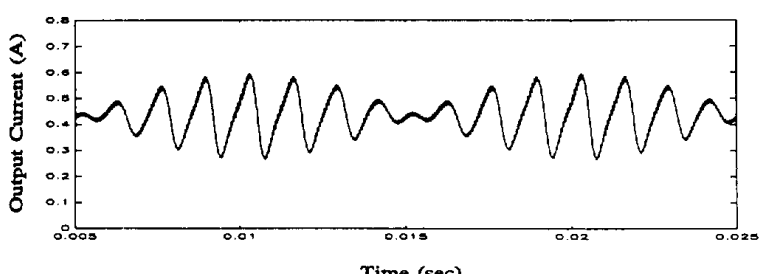

Fig. 6. PSpice simulated output load current waveform of boost amplifier.

Different to the buck converter, $K_{4}$ in (17) is not zero for the boost converter, which implies that the interaction between the tones of $\tilde{\delta}$ as well as the interaction between the tones of $\tilde{\delta}$ and $\tilde{v} g$ exist. Thus the frequency band of the spectrum as shown in Fig. 5(b) or (c) is much wider than that for the buck converter.

Moreover, the boost converter can be used as an amplifier by translating perturbations in the control signal into voltage excursions at the converter output while maintaining the supply line voltage constant. By using the same boost amplifier as that of [8], the steady-state output load current waveform resulted from the PSpice simulation is shown in Fig. 6. Thus the FFT and the VFS spectral components are compared with the experimental results obtained in [8]. As seen in Table VI, the agreement is good.

\section{PWM Buck-Boost Converters}

Since it is interesting to apply the proposed approach to other commonly used converter topologies, the approach is further applied to the PWM buck-boost converter as shown in Fig. 3(c). Also, in order to show the validity of the resulted model under other switching frequencies, the converter is operated at $20 \mathrm{kHz}$ while its component values and largesignal perturbations are the same as those of the buck and the boost converters. Following the previous procedure, the steady-state output voltage waveform is shown in Fig. 7(a), the FFT spectrum is plotted in Fig. 7(b), the VFS spectrum is plotted in Fig. 7(c), and the significant spectral components are tabulated in Table VII. Again, the agreement is very good. 
TABLE VII

SPECtral Components of Buck-Boost Converter Output Voltage

\begin{tabular}{lll}
\hline Frequency $(\mathrm{Hz})$ & FFT Magnitude $(\mathrm{dB})$ & VFS Magnitude $(\mathrm{dB})$ \\
\hline 0 & 0 & 0 \\
200 & -14.0 & -13.9 \\
300 & -14.1 & -13.9 \\
500 & -22.9 & -23.2 \\
700 & -8.3 & -8.2 \\
800 & -8.5 & -8.4 \\
1000 & -23.5 & -23.7 \\
1400 & -22.3 & -22.4 \\
1500 & -17.7 & -17.4 \\
1600 & -23.3 & -23.4 \\
\hline
\end{tabular}

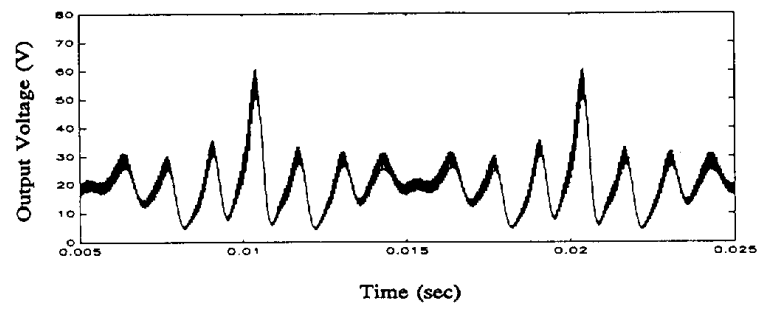

(a)

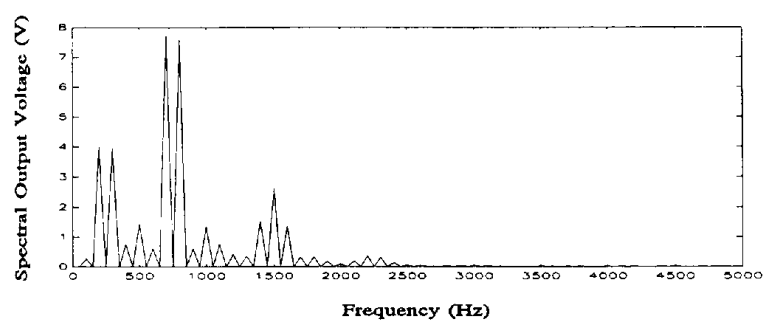

(b)

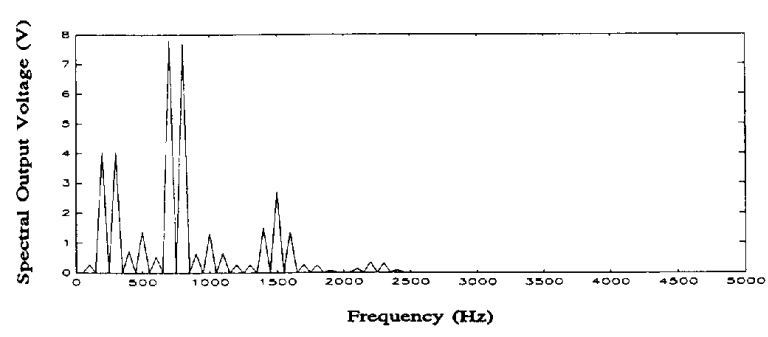

(c)

Fig. 7. Output voltage of buck-boost converter. (a) Waveform using PSpice. (b) Spectrum using FFT and (c) spectrum using VFS.

Similar to the boost converter, $K_{4}$ in (17) is not zero for the buck-boost converter. Thus the frequency band of the spectrum as shown in Fig. 7(b) or (c) is much wider than that for the buck converter.

\section{CONCLUSION}

The spectral modeling of power converters has been proposed and verified. The modeling approach is to newly extend the Volterra functional series to nonlinear multiinput systems, such as PWM converters with control signal and supply line inputs. The spectral model of PWM converters has been developed in a closed form, which is very useful for computeraided spectral analysis. Thus, by adjusting proper system parameters, one can minimize certain spectral components which may be harmful to the system.

The modeling approach has been successfully applied to various PWM converters including the buck, the boost, and the buck-boost topologies. It can be found that the buck converter has a relatively narrow frequency band of output voltage spectrum while the frequency band of the boost or the buck-boost converter is much wider, which can be accurately predicted by the proposed approach. Finally, the approach is so general that it can readily be extended to other power conversion systems.

\section{ACKNOWLEDGMENT}

The authors would like to thank Dr. K. M. Tsang for many informative discussions.

\section{REFERENCES}

[1] K. T. Chau, Y. S. Lee, and A. Ioinovici, "Computer-aided modeling of quasi-resonant converters in the presence of parasitic losses by using the MISSCO concept," IEEE Trans. Ind. Electron., vol. 38, pp. 455-462, 1991.

[2] C. C. Chan and K. T. Chau, "A fast and exact time-domain simulation of switched-mode power regulators," IEEE Tran. Ind. Electron., vol. 39 , pp. 341-350, 1992.

[3] R. D. Middlebrook and S. Cuk, "A general unified approach to modelling switching converter power stages," in Proc. IEEE Power Electron. Specialists Conf., 1976, pp. 18-31.

[4] R. Tymerski, V. Vorpérian, F. C. Y. Lee, and W. T. Baumann, "Nonlinear modeling of the PWM switch," IEEE Trans. Power Electron., vol. 4, pp. 225-233, 1989.

[5] J. J. Bussgang, L. Ehrman, and J. W. Graham, "Analysis of nonlinear systems with multiple inputs," Proc. IEEE, vol. 62, pp. 1088-1119, 1974.

[6] L. O. Chua and C. Y. Ng, "Frequency domain analysis of nonlinear systems: General theory," IEEE Trans. Circuits Syst., vol. 3, pp. 165-185, 1979 .

[7] M. Schetzen, The Volterra and Wiener Theories of Nonlinear Systems. New York: Wiley, 1980.

[8] R. Tymerski, "Volterra series modeling of power conversion systems," IEEE Trans. Power Electron., vol. 6, pp. 712-718, 1991.

[9] K. T. Chau, "Spectral Analysis for power electronics systems," Dep. Elec. Eng., Hong Kong Polytechnic, Tech. Rep. PE 91-2, 1991.

[10] V. Volterra, "Sopra le funzioni che dipendono de altre funzioni," Rend. R. Academicia dei Lincei $2^{\circ}$ Sem, pp. 97-105, 141-146 and 153-158, 1887.

[11] - Theory of Functionals and of Integral and Integro-Differential Equations. Dover, 1959.

[12] N. Wiener, "Response of a nonlinear device to noise," Radiation Laboratory, M.I.T., Report 129, 1942.

[13] J. F. Barrett, "The use of functionals in the analysis of nonlinear physical systems," J. Electron. Contr., vol. 15, pp. 567-615, 1963.

[14] S. A. Billings, "Identification of nonlinear systems-A survey," Proc. IEE, vol. 127, part D, pp. 272-285, 1980.

[15] S. Narayanan, "Application of Volterra series to intermodulation distortion analysis of a transistor feedback amplifier," IEEE Trans. Circuit Theory, vol. 17, pp. 518-527, 1970.

[16] T. K. Sarkar and D. D. Wiener, "Scattering analysis of nonlinearly loaded antennas," IEEE Trans. Antennas Propagation, vol. 24, pp. $125-131,1976$

[17] W. J. Lawless and M. Schwartz, "Binary signaling over channels containing quadratic nonlinearities," IEEE Trans. Commun., vol. 22, pp. $288-297,1974$.

[18] F. Fallside and J. Waddington, "Exact and Volterra analysis of synchronous machine stability," Int. J. Contr., vol. 11, pp. 199-211, 1970. 
[19] H. D. Hogge and W. C. Meechan, "The Wiener-Hermite expansion applied to decaying isotropic turbulence using a renormalized timedependent base," J. Fluid Mechanics, vol. 85, pp. 325-347, 1978.

[20] E. D. Lipson, "White noise analysis of phycomyces light growth response system I, II, II," Biophys. J., vol. 15, pp. 989-1045, 1975.

[21] P. Z. Marmarelis and V. Z. Marmarelis, Analysis of Physiological Systems-The White Noise Approach. New York: Plenum, 1978.

[22] A. F. Witulski and R. W. Erickson, "Extension of state space averaging to resonant switches and beyond," IEEE Trans. Power Electron., vol. 5, pp. $98-109,1990$.

[23] M. H. Rashid, SPICE for Circuits and Electronics using PSpice. Englewood Cliffs, NJ: Prentice-Hall, 1990.

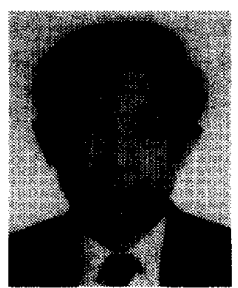

C. C. Chan (F'92) started his professional electrical engineering career in 1959. He has been working 11 years in industry and 24 years in academic institutions. He is presently the endowed Honda Professor of Engineering and Director of the International Research Center for Electric Vehicles, University of Hong Kong. He was visiting professor at several well known universities, including the University of California at Berkeley. He serves as Consultant to several organizations in Hong Kong and the U.S.A. His research interest is in advanced motor drives and electric vehicles. He has published four books and over 80 technical papers on electrical engineering.

Dr. Chan is a Fellow of the IEE and HKIE, Chairman of the IEEE Technical Committee, and is listed in International Leaders of Achievement, Men of Achievement, Who's Who in Australasia and the Far East, etc. $\mathrm{He}$ is the co-founder of the World Electric Vehicle Association.

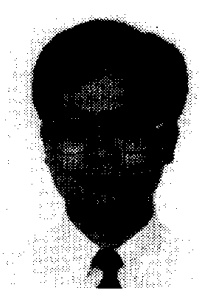

Kwok-Tong Chau (M'89) received the first-class honours B. Sc.(Eng.), M. Phil., and Ph.D. degrees all in electrical and electronic engineering from the University of Hong Kong in 1988, 1991, and 1993, respectively.

Since 1990, he has been with the Hong Kong Polytechnic, where he currently works as Lecturer in the Department of Electrical Engineering. His research interests include power electronics, circuits and systems, and electric drives. He has published over 30 refereed technical papers and several industrial reports.

Dr. Chau is also the recipient of the Sir Edward Youde Memorial Fellowships in 1988-1990 and 1989-1990. 